\title{
Fundamental Study on Intraoperative Quantification of Gastrointestinal Viability by Transmission Light Intensity Analysis
}

\author{
Yoshitaka Minami ${ }^{1}$, Takashi Ohnishi ${ }^{2}$, Hiroshi Kawahira ${ }^{2}$, and Hideaki Haneishi ${ }^{2}$ \\ ${ }^{1}$ Graduate School of Engineering, Chiba University, Chiba, Japan \\ 1-33 Yayoi-cho, Inage-ku, Chiba, 263-8522 Japan \\ 2 Center for Frontier Medical Engineering, Chiba University \\ \{y_minami,t-ohnishi\}@echiba-u.jp, \\ $\{$ hk, haneishi\}@faculty.chiba-u.jp
}

\begin{abstract}
In gastrointestinal surgery, surgeon subjectively judges if the organ is healthy from the color. However it is difficult to discriminate a small difference of organ's color by visual inspection. In this paper, we focus on the tissue oxygen saturation $\left(\mathrm{StO}_{2}\right)$ that represents balance of oxygen demand and supply in tissue and try to estimate its value by transmitted light intensity analysis. We developed a system for measurement of transmitted light intensity using a compact spectrometer and a halogen light source and collected transmitted light intensity data from pig's small intestines. Absorbance of the tissue was then calculated from those data. On the basis of Beer-Lambert law, we estimated $\mathrm{StO}_{2}$ from the calculated absorbance. Results of evaluation experiment to pig's small intestines suggested the possibility of quantitative evaluation of tissue viability by the proposed method.
\end{abstract}

Keywords: Transmitted light intensity analysis, Tissue oxygen saturation $\left(\mathrm{StO}_{2}\right)$, Beer-Lambert law.

\section{Introduction}

In the reconstructive and resection surgery of the hollow organ such as small intestine the determination of the viability is very important. This determination is performed by visual inspection based on the organ's color. However it is difficult to discriminate a small difference of organ's color. For this reason, quantification of gastrointestinal viability is required.

As solution for this problem, quantification methods for gastrointestinal viability using of spectral information have been studied. In those works, a hyper spectral camera or a combination of LED light source and color CCD camera were used. Among those studies, H. Akbari et al[1] and E. Kohlenberg et al[2] segmented the ischemic site by focusing on the differences of the spectral reflectance between the ischemic intestine and normal intestine. Other groups[3]-[5] presented estimation methods of oxygen saturation based on reflected intensity analysis. In these studies, improving the accuracy of determination is difficult due to the difference of the optical path and 
the depth of light invasion caused by the scattering in the tissue. On the other hand, quantitative evaluation based on blood flow measurement using a laser Doppler velocimeter[6] is not practical because this is time-consuming method and the influence of motion artifacts is large. Although a pulse oximeter can measure the oxygen saturation [7], it is necessary to detect the beating, and thus the measurement site is limited.

In this paper, we propose a quantitative evaluation method of viability based on transmitted light intensity analysis that is relatively insulated from the influence of scattering in tissue. As an indicator of quantitative evaluation of blood circulation, we focus on the tissue oxygen saturation $\left(\mathrm{StO}_{2}\right)$ that represents balance of oxygen demand and supply in tissue.

In this attempt, we developed a system for measurement of transmitted light intensity by prototyping a compact probe. Furthermore, in-vivo measurement with this system was performed using pig's small intestines. Absorbance of the tissue was then calculated from the measured data. On the basis of Beer-Lambert law, we defined the organ model formula and estimated $\mathrm{StO}_{2}$ from the calculated absorbance.

\section{Construction of the Measurement System and Data Collection}

\subsection{Measurement System}

As a light emitting device, we used halogen light source (MHF-G150LR, MORITEX) having a broadband emission characteristics from visible to near-infrared regions. Fig. 1 shows the trial product of compact probe. Compact spectrometer (USB2000, Ocean Optics) was used as a light receiving device. A light guide connected to the halogen light source was placed on the bottom and the compact spectrometer probe was placed on top so as to align their optical axis. Organ is clipped by the compact probe and the transmitted light intensity is collected.

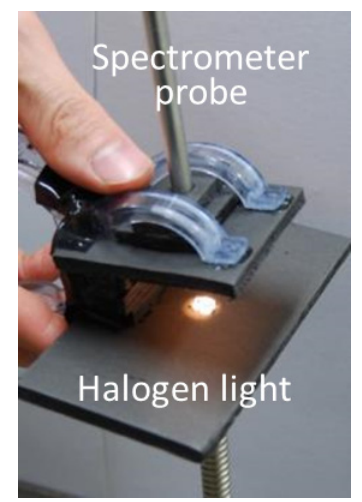

Fig. 1. Trial Product of Compact Probe 


\subsection{Collection of Absorbance Data}

In order to collect transmitted light intensity of intestine in various blood circulation states, animal in-vivo experiments with pig were performed. The trial product of compact probe was used. The wavelength region from 600 to $950 \mathrm{~nm}$ at $5 \mathrm{~nm}$ intervals was used.

First, incident light intensity was collected without clipping intestine to compact probe. Next, we opened the abdominal cavity and clipped intestine to compact probe. Transmitted light intensity of 15 healthy small intestines were collected. At this collection, we also measured oxygen saturation of large vessel region with pulsation by a pulse oximeter. The measured region was near the site where we collected the transmitted light intensity. We define the measured value by the pulse oximeter as a reference. Thereafter, we create the poor blood circulation state by ligating the small intestine and continued to collect transmitted light intensity from 4 intestines for 3 minutes at 20 seconds intervals after ligation. Fig. 2(a) shows the environment of the animal experiment, and Fig. 2(b) shows the process of ligation.

Absorbance $A_{\text {organ }}(\lambda)$ of intestine is calculated from the collected data as

$$
A_{\text {organ }}(\lambda)=\log \left(\frac{I_{\text {in }}(\lambda)-I_{\text {dark }}(\lambda)}{I_{\text {out }}(\lambda)-I_{\text {dark }}(\lambda)}\right) .
$$

Here $I_{\text {in }}(\lambda)$ is incident light intensity, $I_{\text {out }}(\lambda)$ is transmitted light intensity, and $I_{\text {dark }}(\lambda)$ is dark current intensity. Fig.2 (c) shows some examples of the calculated absorbance.

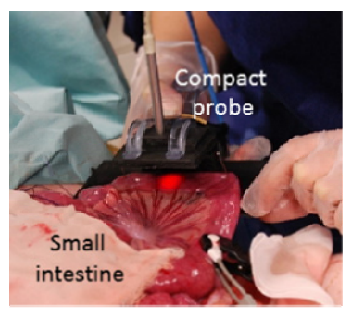

(a)

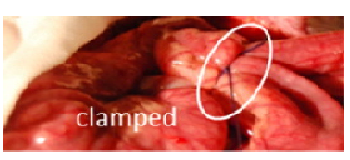

(b)

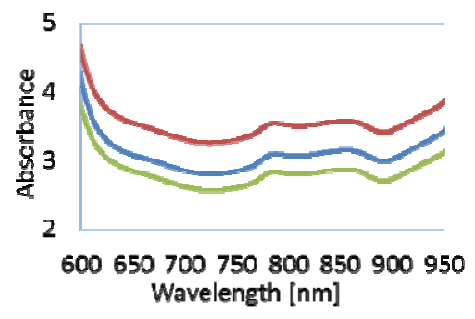

(c)

Fig. 2. Environment of Animal Experiment. (a) state of collecting data, and (b) state of ligation. (c) some examples of the absorbance.

\section{Method for Estimation of Tissue Oxygen saturation}

\subsection{Modeling of the Absorbance Characteristics in Organ}

Estimation of the tissue oxygen saturation is based on the Beer-Lambert law. In this law, when the $n$ light-absorbing substances exist in the tissue, total absorbance $A(\lambda)$ is the sum of each absorbance, and given by Eq. (2). 


$$
A(\lambda)=\sum_{i=1}^{n} \varepsilon_{i}(\lambda) C_{i} l
$$

where $\varepsilon_{i}(\lambda)$ and $C_{i}$ denotes the extinction coefficient and the concentration of $i$-th lightabsorbing substance, respectively. $l$ denotes the optical path length of the tissue.

In fact, the value calculated by Eq. (1) consists of not only blood absorbance but also the other tissue absorbance and scattering factor. It is considered that there are two kinds of light-absorbing substance in organ. One is blood components consisting of oxygenated hemoglobin and deoxygenated hemoglobin. The other is components such as muscle, nerve or fat that does not contribute the variation of oxygen saturation. Thus, we define the organ absorbance $A_{\text {organ }}(\lambda)$ by adding the correction term $A_{\text {tissue }}(\lambda)$ that represents absorption and scattering of the other tissues to the blood absorbance $A_{\text {blood }}(\lambda)$ as

$$
A_{\text {organ }}(\lambda)=A_{\text {blood }}(\lambda)+A_{\text {tissue }}(\lambda) .
$$

Here the absorbance of blood is given by Eq. (4) using oxygenated and deoxygenated hemoglobin extinction coefficient $\varepsilon_{\mathrm{o}}(\lambda)$ and $\varepsilon_{\mathrm{d}}(\lambda)$, concentration $C_{\mathrm{o}}$ and $C_{\mathrm{d}}$ as

$$
A_{\text {blood }}(\lambda)=\varepsilon_{\mathrm{o}}(\lambda) C_{\mathrm{o}} l+\varepsilon_{\mathrm{d}}(\lambda) C_{\mathrm{d}} l .
$$

If we can estimate concentration $C_{\mathrm{o}}$ and $C_{\mathrm{d}}, \mathrm{StO}_{2}$ is given by

$$
\mathrm{StO}_{2}=\frac{C_{\mathrm{o}}}{C_{\mathrm{o}}+C_{\mathrm{d}}} \times 100
$$

Since the oxygenated and deoxygenated hemoglobin extinction coefficient $\varepsilon_{0}(\lambda)$ and $\varepsilon_{\mathrm{d}}$ ( $\lambda$ ) are already known, $\mathrm{StO}_{2}$ can be obtained if $A_{\text {blood }}(\lambda)$ and $l$ are estimated. Estimating $l$ is not a difficult task because the intestine is clipped and the thickness can be measured. The problem is how to estimate $A_{\text {tissue }}(\lambda)$. From the observation of measured data, we consider that it can be represented by a small number of principal components of spectral absorbance. Actual estimation technique is described below.

\subsection{Estimation Method of Tissue Oxygen Saturation}

Based on the above-mentioned model, we propose an estimation method of tissue oxygen saturation. It consists of two steps: preparation step and estimation step.

In the preparation step, we use some well-controlled samples of organ for learning and determine principal components to represent $A_{\text {tissue }}(\lambda)$ from the measured data. More specifically, organ absorbance $A_{\text {organ }}(\lambda)$ is directly measured. On the other hand, we measure concentration values by a blood analyzer and estimate $A_{\text {blood }}(\lambda)$ based on Beer-Lambert law. From these two values, tissue absorbance is calculated from Eq. (3) as 


$$
\hat{A}_{\text {tissue }}(\lambda)=A_{\text {organ }}(\lambda)-A_{\text {blood }}(\lambda) \text {. }
$$

This estimation is performed for $N$ examples. Then principal component analysis is applied to those data. Using the mean component $u_{\mathrm{m}}(\lambda)$ and principal components $u_{\mathrm{i}}(\lambda)$ that show sufficient contribution ratio, we determine $A_{\text {tissue }}(\lambda)$ as

$$
A_{\text {tissue }}(\lambda)=\sum_{i=1}^{n} \alpha_{i} u_{i}(\lambda)+\alpha_{m} u_{m}(\lambda)
$$

Here $n$ is number of used principal components and $\alpha$ is coefficient. Using this function, we defined organ model formula as

$$
A_{\text {organ }}(\lambda)=\varepsilon_{\mathrm{o}}(\lambda) C_{\mathrm{o}} l+\varepsilon_{\mathrm{d}}(\lambda) C_{\mathrm{d}} l+\sum_{i=1}^{n} \alpha_{i} u_{i}(\lambda)+\alpha_{0} u_{\mathrm{m}}(\lambda)
$$

For the target organ, we substitute organ absorbance data collected intraoperatively into the model expression. Then, we apply multiple regression analysis to the model formula and calculate regression coefficients including $C_{\mathrm{o}} l, C_{\mathrm{d}} l$.

\section{Experiment for Estimation of Tissue Oxygen Saturation}

\subsection{Estimation Experiment to Healthy Intestines}

The proposed method was applied to 15 absorbance data of healthy intestines of two pigs. Determination of the model and the estimation of $\mathrm{StO}_{2}$ were conducted by the Leave-One-Out technique. Namely, determination of the basis functions of $A_{\text {tissue }}(\lambda)$ was done using 14 absorbance data out of 15 original data and the remaining one data was estimated. In PCA, the first and second principal components were used because they showed enough cumulative contribution ratio. Fig.3 (a), (b) show an example of principal components and mean component, respectively.

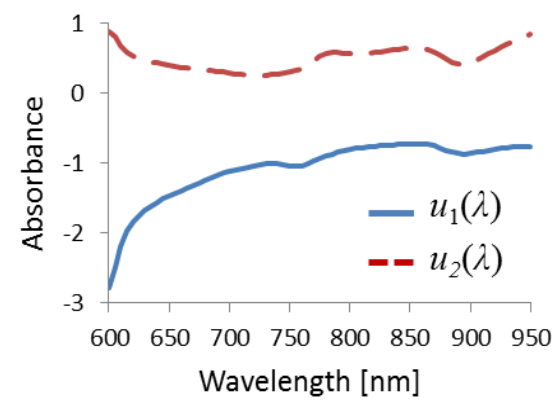

(a)

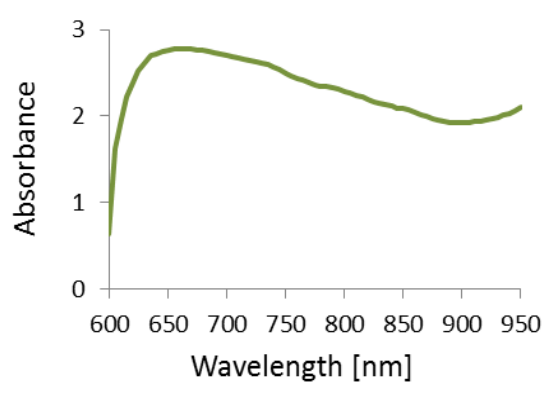

(b)

Fig. 3. A sample of used principal components. (a) is the $1^{\text {st }}$ and $2^{\text {nd }}$ principal component. (b) is mean component. 
We estimated $\mathrm{StO}_{2}$ using the model formula and compared the estimated value and the reference value by a pulse oximeter. Wavelength range from 600 to $950 \mathrm{~nm}$ at 5 $\mathrm{nm}$ intervals was used for multiple regression analysis.

Fig. 4 shows the result. For intestines that have different blood circulation states, $\mathrm{StO}_{2}$ was estimated with $2.0 \%$ of average error and $4.6 \%$ of maximum error.

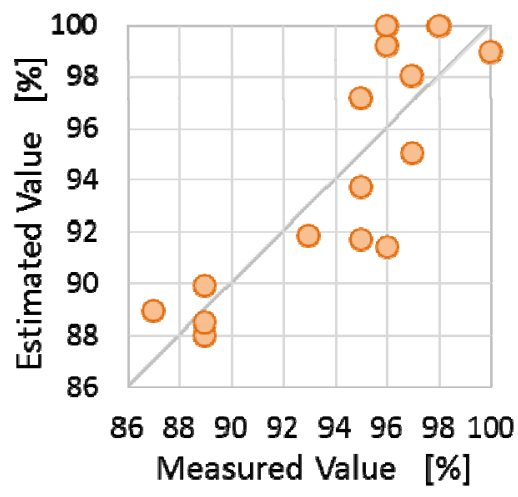

Fig. 4. Results of $\mathrm{StO}_{2}$ Estimation to Healthy Intestines

\subsection{Estimation Experiment to Ligated Intestines}

The proposed method was applied to time-dependent absorbance data of ligated intestines. We estimated tissue oxygen saturation of 4 intestines for 3 minutes at 20 seconds intervals after ligation. The model formula we used was the same as that of the experiment for healthy intestines.

Fig. 5 shows the result. For all time-dependent absorbance data of ligated intestines, we confirmed the decrease of tissue oxygen saturation. That is, possibility of intraoperative monitoring of changes in blood circulation state of small intestine was suggested.

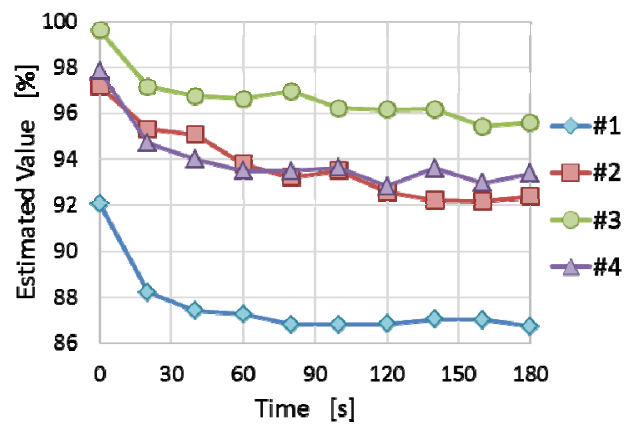

Fig. 5. Results of $\mathrm{StO}_{2}$ estimation of clamped small intestine 


\section{Conclusion}

We made constructing a transmitted light intensity measurement system using a compact spectrometer and halogen light source, and collected absorbance data from pig's intestines in in-vivo animal experiments. The data was collected from both of healthy intestines and ischemia ones by ligation.

Based on the Beer-Lambert law, we took into account the absorption and scattering of tissue other than blood and defined organ model formula. Then, using this formula, estimation of $\mathrm{StO}_{2}$ was attempted. $\mathrm{StO}_{2}$ estimation for healthy intestines was performed with $2.0 \%$ of average error and $4.6 \%$ of maximum error. Furthermore, through the experiment of $\mathrm{StO}_{2}$ estimation of ligated intestines, the possibility of intraoperative monitoring of changes in blood circulation state of small intestine was suggested.

Evaluation of the model formula and accuracy improvement by increase of the data with various blood circulation states is future works. Moreover, we will develop a more compact and easy-to-use transmitted light intensity measurement system using LED light source and linear sensor for clinical use.

Acknowledgement. This research was supported by Kakenhi, the Grant-in-Aid for Scientific Research B 25282151 and Chiba University COE start-up program.

\section{References}

1. Akbari, H., Kosugi, Y., Kojima, K., Tanaka, N.: Detection and Analysis of the Intestinal Ischemia Using Visible and Invisible Hyperspectral Imaging. IEEE Transactions on Biomedical Engineering 57(8), 2011-2017 (2010)

2. Kohlenberg, E., Payette, J.R., Sowa, M.G., Levasseur, M.A., Riley, C.B., Leonrdi, L.: Determining intestinal viability by near infrared spectroscopy: A veterinary application. Vibrational Spectroscopy 38, 223-228 (2005)

3. Li, J., Dunmire, B., Beach, K.W., Leotta, D.F.: A reflectance model for non-contact mapping of venous oxygen saturation using a CCD camera. Optics Communications 308, 78-84 (2013)

4. Karliczek, A., Benaron, D.A., Zeebregts, P.C.J., Wiggers, T., van Dam, G.M.: Intraoperative assessment of microperfusion with visible light spectroscopy for prediction of anastomotic leakage in colorectal anastomoses. The Association of Coloproctology of Great Britain and Ireland 12, 1018-1025 (2010)

5. Nishidate, I., Aizu, Y., Mishina, H.: Estimation of melanin and hemoglobin in skin tissue using multiple regression analysis aided by Monte Carlo simulation. Journal of Biomedical Optics 9(4), 700-710 (2004)

6. Urbanavicius, L., Pattyn, P., Putte, D.V., Venskutonis, D.: How to assess intestinal viability during surgery: A review of techniques. World Journal of Gastrointestinal Surgery 3(5), 59-69 (2011)

7. La Hei, E.R., Shun, A.: Intra-operative plus oximetry can help determine intestinal viability. Pediatr. Surg. Int. 17, 120-121 (2001)

8. Tamura, T., Yamakoshi, K., Murakami, H.: Medical Electronic Devices I. CORONA Publishing, Tokyo (2006)

9. Kamiya, A., Ando, J., Masuda, H., Shibata, M., Tsuji, T., Sakuma, I.: Biomechanics of Blood Circulation. CORONA Publishing, Tokyo (2005) 